\section{Zur Lungenchirurgie.}

\author{
Dritte Mittheilung
}

ron

\section{Wilhelm Koch in Dorpat.}

Es suld nun ueun Jahre rerflossen, dass ıch $1 \mathrm{~m}$ Archır für hlinische Chirurgıe ${ }^{1}$ ) eine Versuchsmethode angab), nuttelst deren es unr geluugen war, behelıge Absclinıtte des Lungengewebes, selbst ganze Lappen desselben in Bludegewebe umzuwandeln. Ich verweudete, diesen Zwech zu erreichen, coucentrirte Jodjodkahlosunges, gab au, dass mau die Verwandelung der Lunge zur Narbe auch durch die Galvanocaustik hewrien könne und hol, hervor, dass, be Theren wemırsteus, Eıugriffe der Art fast olune jede Reaction ertragen werden. Eine ähnliche gernge Entpfindlucliheıt auch der menschlichen Lunge schien mir wahrschetulıch, wenn tch an unsere Erfalırungeu über die Lngefälırlıchleıt des Lungenvorfalles und an die un der Regel überaus prompte Heilung der Lungenschiusskanäle dachte. lst es doch uıchts Lnerlıörtes, dass eine Art prıma jutentio selbst dans eintrıtt, wenn die hugel tuberculöses Lungengewebe durchfurchı!

Auf Grund deser Erfahrung trat ich lint dem Vorschlag an die Oeffentluchkeit man möge die ,,chronıschen, noch nicht zu weit vorgeschritteuen" d. h. Localısirten Lungenaffectionen versuchswese auch einmal ınit clururgisshen Waffen bekämpfen. Diese Arbett ist 111 der Neuzeıt die erste gewesen, welche das Princip der Zulässigkelt chururgischer Hulfelesstungen bei jeneu Parenchviulrankihetten der Luuge zu begrunden versuchte, dereu Behandlung bisher ausschliesslich und alleın der inneren Medicin zuerkanut war, eine Thatsache, die ich schon deswegeu betonen muss, weil fast die sämmtlıchen Autoreu, welche nach unır iber diesen Gegenstand sich auscrelassen hraben, mene Benühungen um denselheu zu überselien, fur gut hefindeo.

Iu emer zwetten Arbent ${ }^{2}$ ) entwickelte ıch sodaun zunächıst meine Ansichten uber die besondereu pathologischen Processe, welche un Innern der Luncencaverneu abspielen nussen, wenn anders deren Eriof $\mathrm{f}$ ung und Flächembehandlung erlauht erscheinen solle (starke mit Fieber enhergehende jauchıge Secretion und Blutung). Ich beliauptete weıter ${ }^{3}$ ), ,lass is elu irrationelles Unternelımen ses, localisirtere Plithsen durch eme Flàchenbehandlungs von Caverueu zu behämpfen, welche montteu des erhrankten Gewebes atch zufillıg vorfinden. Hier könve heutzutage entweder nur die Durchsetzung des gesanumten lirabken Pareuchysnıs nut ‘tarli reizenden und desuficureuden Mittelı oder aber die allnıalıge Zertörung dıeses Parenchrmıs, an Besten wohl auf galvanocaustıschen Wege, an Frage honmen. lachdem nan für muige Verwachsunor der benden Plenrablätter Sorge getragen hat".

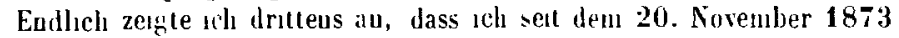
the ,erstere mildore Methode" an elner grosseren Kranhenzall in Anwendungr zu zichen die Gelegeuheit gefunden hätte. Es bezogr sich diese Notiz auf Versuclie, die ich auf der Abthellung des Herrn Geliemrath Frericlıs, unter gütıger Beihilfe der Ilerreu Professoren Ewalı und F. A. Hoffmanu (jetzt Professor in Dorpat) ausführen durfte. Wir brachten mittelst pareuchymatöser Injectiou die reine Jodunctur in Stellen der Lunge, deren tuberculöse Degeneration durch die phisstiallsche Lntersuchung vor Allem aber durch die Acupunctur möglichst sicher bestummt worden war.

Einen ausfülırlichen Bericht öber dıe Resultate dieser Lntersuchungen will xhlt an dieser Stellc schon deswegen uicht geben, weil Herr Stabsarzt Hiller, gegen wartıg Assisteut des Herrn Geheimrath Legden deselben in letzter Zeıt von Neucu aufgenomıen, an einer viel grössereu krankenzalıl als ich durchprobirt und auf's Mannıgfaltigste variirt liat.

Es reuige hervorzulieben, dass die Methode der Acupunctur fast jedesmal jene Plithısen, deren locale Natur wir auf Groud der plıysikitıschen Daagnostıli annelımeu zu müssen neinten, als bereits selır diffuse, inesst über ene gauze Lungeuhälfte verbreitete Processe heunzeiclunete und dass $z$ weitens der Einfluss der Jodtinctur auf die

5) Bd. XV. 1873. S. 706. Ueber die Veränderungen, welche gewisse mechanische und chemisehe Reize in Lungenparenchym hervorbringen.

2) Berliner klinische Wochenschrift 187420 . April, No.16. S. 194. Historisches über die chirurgische Behandlung der Lungencavernen.

3) Berl. klin. Wochenschr. 1sit. 1. (2. \$. 196.
Schrumpfung tuberculös degenerirter Partieen als ein ziemlich irrelevanter sich erwies.

Mein weiterer Plan war uun, die seltenen wirhlich circunıscrıpten tuberculösen Sptzenaffectionen galvanokaustisch zu zerstören uud vor Allem galvanokaustisch auch jene heerdartıgen Processe der Lunge anzıgreıfeu, deren Entstelıung wir uns unabhängig von specifischen Infectionen und nogglich auch bei nıclit dyscrasischen Individuen denkell - die verschiedenen Fornell der Lungengangrän.

Ueber die technische Ausführbarkett und die Berechtigung eines solchen Vorgelıens habe ich mit den Herren Professoren Ewald, Filehue, Hoffnann, Mullk und Senalor in der ersten Hälfte der 70 er Jalıre vielfältıg nuch unterhalten und es war wesentlich nur die Unmöglichkett, passendes Krankenmaterıl im gegebenen Augenblick zu finden, die mich an der Ausführung des Geplanten hinderte.

Es war unter diesen Verhảltosseu für mıch eın besonders glüchlıcher Zuffall, dass hei meııer gegenwärtıgen Anwesenbeit in Berlin auf der línıl des Herrn Geh.-Raths Leyden un der Charité zwè Fälle sıch vorfandeu, welche die Indication zum clururgischen Eungreifen darbotelı. Es laadelte sich um zwei Kranke mit clırdnischer putrıder Bronchitis und Brouchuectasenbildung, welche bei der ïblichen interuen Behandlun: als unluelbar getten nuussten und einem scheren Tode eutgegengiugen. Auch Herr Gelı.-Rath Leyden hatte schon früher iu seiuen klinuschen Vorträgen für derartige Processe die Indicatıon eines operativen Enıgriffs aufgestellt uod be berden Krauken die Operation in Aussıcht genommen. Seine Auflassung stummte durchaus mı derjenıgen äbereın, welche ich auf Grund meiner früheren Untersıchungen vertreten latte. Wir schrıttel daher zur Operatıou beider Fälle, üher deren Methode und Ergelsumse ıch im Folgenden liurz zu bericliten nir gestatte.

Zuvor benutze ichı die Gelegenheit den Herren Stabsärzten der Clıarıté, Dr. Hiller und Dr. Neumann, welche mir bel der Operation und Nachbehandluug guitıst besstanden, mennen bank hiermit auszusprechen.

1. Der 24jährıge Schulumacher Krienlie lat im $\boldsymbol{7}$. Lebensjalıre eine fungöse Erkrankiung des rechteu Knie's überstanden, zwischen dem 9. und 12. Jahre augeblich 4 Mal die Lungenentzündung durclıgemacht und ist von weiteren Lungenentzündungen 1879, 1880, eudlich im Januar 1882 lıemgesucht worden. Vou ilınen lettet er seine jetzt bestelıende Kurzathmokett, seme auffallige Abmagerung, den Verlust des Appetits und die oft reichlıchen Nachtscliweisse ab. Seit 1878 wirft er ell aashaft riechendes Sputum aus, dessen $\mathbf{2 4}$ stïndliche Jenge tu der letzten Zeıt durchıschııttlıch $400 \mathrm{Ccm}$. beträgt.

Die physsikalische Lintersuchung ergiebt die Zeicheu rechtsseltuger Lungenschrumpfung und abnormer Hohlräune 1 in rechten Lnterlappen, wälırend Im Oberldppen nur hatarrl vorlandell scheiut.

Das Sputum besteht aus den behannten 3 Schichteu, es enthält

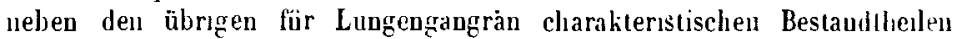
auch Lungengewebsfetzen.

Putrescenz und llenge des Auswurfs ändern sich trotz der 111 allell Varıationen augewendeten Inhalationstherapie gar nicht, eı pleurıtisches Essudat r. u. h. wird zwemal punctirt.

An 26. Jun 1882 wurde die Operation nut der Resection ennes Stücks der sechsten Ruppe begonneu. Der Thermocauter wird, nachden die Verwachsuug beider Pleurablatter erwiesen ist, innutteu der Luugeusubstauz zuu Mediastinum lın allıälig vor çeschobes. Er eroflıet eiue Caverue von der Grösse enter Kinderfaust, in welche, we nas deutlıch sehen hanu, melirere starke Bronchnen luneın mündeo. Diese Caverne ist etwa 3 Querfinger breıt von der Lungenoberfläche eutfernt; eljenso lässt der hüinstlich angelergte trichterförnıge Kanal, welcher durchs Lungengewelse zu ulı lıbeiufulırt, 2-3 Querfinger passiren, so dass also mehrere Quadratzoll des uuteren Lungenlappens zerstört worden sind. Die Reaction nach dem Eiugriff blieb eine nur minımale, die Menge des ausgelunsteten Secrets fiel von 400 auf $120 \mathrm{Ccm}$. pro die.

Die zwette Operation am 30. Juni sollte woniogrıch jene Höhle freilegen, aus welcher das noch immer $120 \mathrm{Ccm}$. betragende Secret sich entleerte. Nach Resection der 8. Rippe etablirt der Thermocauter eine $14 \mathrm{ctm}$. uefe trichterförmige Lungenfistel innerhalb des unteren Lappens in der Richtung nach h. u. und ınnen. Eınıge kleinere Bronchiectasıeen werden zerstölt; doch findet sich kell grösserer Hohlraum, obrohl $z$ wei rom lintersten Ende des Fistelganges aus gegen die Wirbelkörper lin vorgeschobene Pravazspritzen dünnflüssıgen übelrıechenden Eiter aspirıren. Es sitzt also hiuter- und einwärts von der zweiten Lungenfistel entweder ein wandständiges pleurıtisches Exsudat oder aber - ein ettererfüllter grangränöser Heerd.

Die Fretlesung desselben geschalı am 11. Juli durch eine lncision zwischen 8. und 9. Rippe, unterwärts und einwärts rom Angulus scapulae. Thynollösungen, welche von hier aus in den Thorax gelassen werden, gelangen in die Bronchien und werden untermisclit mit deln gaangränösen Sputum sofort expectorirt. -

Auch diese dritte Operation brachte den Auswurf der putriden Massen nucht röllg zum Schwinden. Wir rermuthen noch wetlere Casernen 
im unteren rechten Lungenlappen und gedenken dieselben zu zerstören, sobald wir nuttelst des Aspirationsverfalırens ihren Sitz des Genaueren bestinmt haben. Der Zustand des Patienten ist jetzl ein leidlich befriedigender.

2) Die 29 jährige Handelsfrau Kleiner will in 2. und 7. Lebensjahr Lungenentzündungen, im 15. Jahr einen Typhus und 1878 eine Nephritis durchgemacht haben, nach welcher sich Gelbsucht und ein zeitweise reichlicher, sehr ïbelriechender Auswurf emstellten. Sie wird am 13. Juli 1882 unter den Zeichen thefster Erschöpfung und Macies in die Charité befördert. Hier constatirt man sehr ausgeprägten Icterus, eine frequente dyspnoische Athmung, auflällige Abschwächung der Circulation und eine vollkommene Appetillosigkeit. Der Auswurf aus der Lunge ist exqussit faulıg, dünnflüssig und beträgt in 24 Stunden zwischen 800 und $1000 \mathrm{Ccm}$.

An der rechten Thoraxhälfte beginnt de Däupfung vorn von der 5. Rippe, hinten vom Angulus scapulae; auflallend lautes, deutlıch amphorisches Athmen lässt sich vorn zwischen 5. und 7. Rippe, hinten dicht unter der Scapula nachweisen. Das Rasseln an diesen Stellen klingt metallisclı, die Aspiration liefert vorn und hinten dieselben fötıden Massen, welche ausgehustet werden.

15. Juh 1882 Resection der 6. Rippe auf 4 Zoll Ausdehnung.

Dem Thernocauter wird, entsprechend ener vorher engeführten Prava'schen Nadel de Richtung von fer vorderen Axillarlmue zur äusseru Wand des rechten Herzvorhofes gegelsen. Zwe Zoll von der Lumgenoberfläche dringt er in eine faustgrosse von Trabekeln durchsetzte Caverne en, deren untere Wand zun Thell vom $Z$ werchfell gebildet wrod. Die Trabekeln dieser Höhle werden, so gut es geht, thernocaustısch zerstört, um den Abfluss des Secrets nicht zu hemmen. Fisteln von der Caverne durchs Zwerchfell anr Leber lassen sich nicht nachw'ersen.

Sun wurden zwel nit langen Hohlnadeln arnirte Pravazpritzen vou Jer freiliegenden Caverne mach hinten und oben vorgestossen. Sie aspiriren beide fötıde Massen. Darauf macht der Thermocauter den Wer der Nadeln und gelangt in eme kindskopforosse Höhle, aus welc lier sofort etwa $1000 \mathrm{Ccm}$. fauliger Flüssiglieıt hervorstürzen. Der Zugang zu dieser Caverne wurde so erglebig angelegt, dass drei dicke Cautschoukdraıns ıhn passiren bönnen. Ausspülung sämntlıcher Höhlen mit Thymol und essigsaurer Thonerdelosung.

Die liranke collabirte nach der Operation, bleb indess bus zum 22. Jnlt am Leben. Der Auswirf war wie nut emem Schlarge verschwunden, die Brindschorfe wicheu an den mersten Stellen griten Granulatuoneu.

Was in diesem Falle den todtlichen Alssgang bedingte, war wenger der Engriff als enue phlegmonose Degeneration der Porta hepatis, enne lobuläre Bronchopneumonie des linken untern Lungenlappens um ennen Fremdkorper herum und eme chronische, offenbar schon lange vor der Operation wirksame Septicäme, welche sich unter Anderm anch in feltiger und parenchvimatöser Degeneration der meisten imeren Organe liundrab.

- Herr Gelseimratl Leyden behält sich ror, eıne genauere Besprechung dieser und noch anderer Fälle, welche der Operatıon harren, in semer Zetschrift zu veröffenthchen und dort vor Allem auch die Indicationen des Genaueren zu entwickeln, wellhe rom Standpunlite der inneren Klumk aus das operatıve Einschretten be Lmgengangrän gerechtfertıgt erscheinen lassen.

Ich bemerke an dieser Stelle nur, dass die acut entstandenen, oberflachlich belegenen gangränosen Heerde der lunge nicht selten nach der Pleurahöhle durchbrechen und dann durch eine enfache Incision in die Pleura costalıs nut nachfolgender Ausspülung und eventuell localer Flächenbehandlung der ganoränösen Heerde zur Aushellung gebracht werden können. Fälle dieser Art hat anch Herr Geheimrath Leyden bereits in Strassburg nit Erfolg behandelt; die Tagesliteratur berichtet von Aelınlıchen gerade in der letzten Zeit des Oefteren.

Doch laandelt es sich hier in Grunde genommen doch nicht um Operationen in der Lunge, als vielmehr um die typische Eröffnung der Pleurahöhle. Das Aulsuchen der gangränüsen Höhlen innntten gesunder Lungensulsstanz, die Eröffnung und thelwesse Zerstörung dieser Höhlen und des den Zugang zu ihnen hundernden Lungengewebes stellt Eingriffe dar, dıe un den belden oben erwälnnten Fällen als neu und eıgenthümlich zur Verwendung kamen. -

Bezuglich der Indicationen und namenthch auch der Methodik der thermocaustisclıen Zerstörung bestımmter Lungenabschnıtte, einer Operatıon, die schon heute als relatıv ungefährlich und dabel äusserst wirksam bezeichnet werden kann - bemerke ich noch kurz Folgendes:

Sie möchte ihre Verwendung finden:

1. Beı jener Form der chronischen Lungengangràn, welche aus den sackartigen Bronchiectasieen sich entwickelt und mit der Expectoration grosser Yengen putrider Fliissigkeit vergesellschaftet ist. Findet sich bei der Operation nur eine grössere Höhle, so genügt es, dieselbe durch ene mehr mmier mmlängliche und durchs Lingenparenchyom ge- führte Fistel freizulegen, zu draıniren und ein Paar Tage lang zu irrigiren. Ich habe bet Krlenke gesehen, dass unter einer solchen Behandlung sowohl der Fistelgang als die Cavernen mit schonen Granulationen sich bedeckten und nur wenig absonderten.

Läge die einkainmerige Caverne sehr oberflächlich, so würde schon die einfache Incision resp. die Enführung eines dicken Doppeltroicarts in sie hineın mit nachfolgender Irrigation zur Herbeiführung der Heilung genügen. - Handelt es sich aber um jene Fälle, in denen viele kleınere Bronchiectasieen unter emander conmunıcıren, so möclite es angezeigrt sein, diese von aussen geöffneten vıelbuchtıgen Höhlen in einen grösseren Hohlraum zu verwandeln. Dse Nachbohandlung ist grenau die vorhın erwähnte.

2. Liegt enne acute Lungengangrän vor, bei welcher, we das beispielsweise bei Schussverletzungen vorkommt, abgestorbenes und faulges Gewebe noch ntcht sequestrirt und ausgestossen, sondern ron ödematösem hepausirtem Lungengewebe unfasst ist, so mochte neben der Fistelbıldung sofort auch noch die möglıchst vollständige Verschorfung der lirandigen Lungenpartie vorgenonmen werden missen.

Die Bildung eines trichterförmugen Substanzverlustes in den Lungen scheint mir

3. dann indicırt, wenn in die Bronchien gerathene Fremdkörper auf dem natürlıchen Wecre lucht eliminirt werden sondern zu Bronchoblennorrhoeen und Enschinelamig des benachbarten Lungenjarenchyms Veranlassung geben. Ausnalmuswelse dürfte duch luer die einfacle Incision geniigen.

Dahingestellt muss uch es in . Ingenblick lassen, ob dhe galvanokaustische Zerstörungr elnes unfanglicheren Lungenstüclies

4. bei jener Form der fötiden und putrulen Bronchitis, Jueı welcher Bronchtectasieen sich nucht nachweisen lassen

5. endlich bes der jedenfalls seltenen Form ganz localistrter Lumgentuberculose

seıne Wirksamlieıt bewähren wird.

Es wäre denkbar, dass bet 4. wenigstens allem die Durchsetzung des Lungengewelbes mit desinficirenden und secretionshemmenden Medicamenten passende Verwendung finden bonnte. -

Die galvanoliaustische, bald nur eine Lungenfistel etablirende, bald die kranken Lungenabschntte total zerstörende Operatıon hat jedenfalls das Bestreben zu entfalten, von der athmenden Substanz nüglichst viel zu erlılten. Sur unter stetıger Berücksichtıgung dieser Vorsıchı möchte es moghch seu, z. B. anch jene trar nicht so seltenen Fälle zur Heilung zu bringen, wo ale bronchnectatıschen Heerde zerstreut entweder über die verschiedenen Lappen der emeu Lungenlaalfte oder gar in belden Lungen sich finden. Lie Voralssetzung, an welche die Ausfïlirbalkett emer solchen Forderung stch hnüpft, 1st abel, dass die derenerirten Heerde vor der Operation auf's Genaueste localisirt werden. Hierzu genïgt hekannthch die physlialische Diagnostik, we ste heute geuht ward, nicht. Sie reicht biswellen ja nicht einmal ho. um zu bestummen. ob de Gangràn in ıler rechten oder linkeu Lungenlälfte sich belindet. Hıe trilt helfend das bisher micht abrewendete Aspirationsverfahren en. Man armurt eine Pravaz'sche Spritze nut Hohlnateln, die man in der Länge von $7-15 \mathrm{Ctm}$, vorràthg halten muss, führt diese clurch lie Haut in die Lungensubstanz ein und andert in systematischer Aufeluamderfolce die Richtung der Nadelspitze so lange lis die Aspuration das putrude Secret in reichlicher Menge zum Spritzenbirner hinen befordert.

lch und namentloch Heri Stabsarzt Hiller haben dieses Verfahren, das bet Fremdhörpern und Tuberculose dureh die empfache Acupunctur ersetzt werden kann, hunderte Male ohne ircend einen Sachthenl für die Kranken in Verwendung gezogren. Warnm man mit der Acupmuctur, we mit der Ispiration be ganz oljerflachlichen Cavernen, die man nicht sofort operiren will, vorsichtig sem muss, wollen vir Beide spater das Genauere mutheilen. -

Da rh die Indolenz der Lunge gegen mechanische, chemsche und thermische Reize schon 1872 und 1873 kannte, da ich in dieser Zeit bereits die narbige Schrumpfung ganzer Lungenlappen herbenuführen verstand, la ich endhch wusste, mit welcher Leichtigkelt emerselts rorgefallenes Lungengewebe sich sequestrirt. andrersents fremde itcht faulige von aussen her in die Lunge enncefuhrte fiörper in thr abseliapselt werden - han wh auch schon früh auf den Gedanken, die Lunge in Stücken und lappenweise zu exstirpiren. Es lhut Nichts zur Sache, dass ich dererle Versuche ebenfalls berelts 1872 ausführte; lch stand von hirer Mitthetungr $11 \mathrm{~m}$ so williger ab, als nach mener Memung die Lungenresection am Menschen eine nennensnerthe Zuhunft alcht haben liann.

Beim Lungenvorfall wäre die Resection eine Luxusoperation, da wir wissen. das der Vorfall so gar leıcht emschrmmpft, andererseits es Phlicht ist, dse athmende Obertäche durch fribzeticte Reposition des Vorfalles so gross wit möglich zu erhaltell.

Kommen aber Ilcerationen nul fremde liorper herum und die verschiedenen Formen der Bronchectasieen zur Behandlung, danu opfert die Exsturpation selbstredend in den allermesten Fälen elsenfalls zu viel: sle 
wird geradezn illusoristh, wenn die fanlenden Heerde anf beiden Seiten oder in den verschiedenen Lappen enuer Sente uisten.

Lud niber die Anssichten der Methode, die Tnberculose der Lnnge zn beseltıgen, wird maan angesıchts der Erfahruagen, welche die Chirurgen in den letaten Jahren bes der Ausrottnng anderer tuberculöser Organe geniacht haben, überschwảnglichen Hoffunggen zur Zeıt sich ebenfalls uıcht hingeben dürfen.

lch meıne, dass in diesem Pnnkte die Chancen für das Mlesser ebeuso ungüuslı liegen, wıe für den Thermocauler, dass aber, wenn einmal resecurt werden soll, die Verkolulung der kranken Theile de Schwierigbeat nugeht, auf lie Lisung der bekanullich oft ülerans festen Vern wachsongen zwischen Lunge, Thorax und Medastinum lange Zeit verwenden zu müsseu.

Zuw Schluss deute ich an, dass ich be1 Schnssverletznngen der Lunge und glemcheetıger starlier Blutung ans Ilır, nach voranfgehender Rippenresection die Lunģenwnrzel herrorziehıen nod dem Gesichl zugänglich machen wirde. Gelange die localısirte Unterbıdung des eroffneten Gefasses micht, so müsste die Imstechung des blutenden Theiles versncht werden. Nach der Rücklagernng der Lunge würde eine prophylactische Dranage des pleuralen Ranmes nnter eınem autıseptischen Verbande hier we 111 jenen seltenen Fällen vou Lungenbrand zu versuchen seın, bei welchen die Verklebnng zwischen den beiden pleuralen Blättern nicht erriebıg genug ansfiel. 